\title{
Nocardiosis in anaemic patients given steroids
}

\author{
D. N. WhitMore, G. A. GRESHAM, AND M. J. GRAYSON \\ From the Department of Pathology, University of Cambridge, and Addenbrooke's Hospital, Cambridge
}

SYNOPSIS Two fatal cases of infection with Nocardia asteroides are described with necropsy findings in one. Both patients had been anaemic and had received steroid therapy.

The bacteriological problems of species recognition in the genus Nocardia are considered and the results of infection in experimental animals described.

Nocardia asteroides rarely produces human infection in Britain although the infection is not uncommon in the United States of America (McQuown, 1955). In the majority of such cases pulmonary lesions occur and the respiratory tract is assumed to be the portal of entry (Ballenger and Goldring, 1957). Infection may be disseminated to the brain causing cerebral abscesses; Nocardia asteroides was first isolated from a cerebral abscess by Eppinger in 1890.

Two cases are reported; in both the mode of infection is debatable. The patients suffered from severe anaemia and were treated with steroids; these may have been predisposing factors.

\section{CASE REPORTS}

CASE 1 A man aged 73, a retired hedger, was admitted in July 1958 with nine months' history of jaundice and fatigue. He was very pale, slightly jaundiced, and the spleen was moderately enlarged.

On admission $\mathrm{Hb}$ was $6.0 \mathrm{~g}$. per $100 \mathrm{ml}$. of blood. Reticulocytes were $29 \%$ and W.B.C. 8,500/c.mm. (polymorphs 6,800, lymphocytes 1,300 , monocytes 400 ). The plasma bilirubin level was $1.9 \mathrm{mg} . / 100 \mathrm{ml}$. The blood group was $\mathrm{O} R \mathrm{R}$ positive. The sternal marrow showed marked hyperplasia of the erythroid series. The direct sensitization test (Coombs) was positive and a 'cold' antibody was found in the serum.

A diagnosis of acquired haemolytic anaemia of the 'autoimmune' type was made and prednisone, $15 \mathrm{mg}$. t.d.s., started. The haemoglobin rose steadily to $10.6 \mathrm{~g}$. per $100 \mathrm{ml}$. in approximately four weeks and the patient was discharged on prednisone, $25 \mathrm{mg}$. daily.

Three months after the first admission he presented with painful swellings of the left loin and shoulder which appeared to be acute pyogenic abscesses. The temperature was $102^{\circ} \mathrm{F}$. and the W.B.C. $24,000 /$ c.mm. with a polymorphonuclear leucocytosis. Both abscesses were incised; that in the left loin extended between the muscle layers of the abdominal wall into the left peripheric fat; the abscess in the left shoulder was limited to the subcutaneous tissues. Several ounces of thick yellow pus were drained from both abscesses. Smears of pus showed a Gram-positive filamentous organism subsequently identified as Nocardia. Chloramphenicol, $1 \mathrm{~g}$. daily, was given for a week. Further abscesses appeared in the subcutaneous tissues of the right calf and left thigh; these were incised and cultures of the pus grew Nocardia. Treatment with sulphadiazine, $4 \mathrm{~g}$./day, was begun and continued until the patient's death.

The haemolytic process appeared to escape control with the appearance of the first abscess, the blood haemoglobin fell to $6 \mathrm{~g}$. per $100 \mathrm{ml}$., and there was only transient improvement with blood transfusion and an increased steroid dosage. Five months after the second admission another abscess in the left loin was drained. Proteus vulgaris, Bact. coli, and non-haemolytic streptococci were grown from the pus; Nocardia was not isolated. Shortly after this the patient had a series of generalized convulsions. Examination of the central nervous system showed no abnormality; the cerebrospinal fluid was normal.

Deterioration continued, the haemoglobin fell to $4 \cdot 5 \mathrm{~g}$. per $100 \mathrm{ml}$., and the patient died eight months after he was first admitted.

Necropsy (P59/191) An abscess in the left psoas, $2 \mathrm{~cm}$. in diameter contained yellow-grey material with much surrounding fibrosis.

A thin-walled abscess $(3 \mathrm{~cm}$. diameter) was present immediately to the right of the superior sagittal sinus, $3 \mathrm{~cm}$. from the confluence of sinuses. The superior sagittal sinus was obliterated in the region of the abscess by organizing thrombus. Section of the brain showed two abscesses in the right fronto-parietal region.

The heart was of normal size; the cardiac muscle showed tabby-cat striation. Grey-yellow vegetations (up to $0.5 \mathrm{~cm}$. diameter) were present on the atrial aspects of the mitral and tricuspid valves and the edges of the aortic valve cusps were thickened by similar vegetations. 
The spleen was moderately enlarged $(320 \mathrm{~g}$.), with a soft purple cut surface. A piece of spleen turned blue in acid ferrocyanide solution. The marrow was red and gelatinous in appearance and extended as far as the middle of the right femur; similar marrow was present throughout the vertebral column, and was increased in the diploe of the skull. Lymph nodes were not enlarged.

The kidneys showed no significant abnormality other than sulphonamide crystals in the renal pelves; these were also seen in the bladder.

The right pleural cavity contained $100 \mathrm{ml}$. of cloudy yellow fluid; the lungs were oedematous but showed no evidence of infection or of a source for the nocardial infection.

The liver was slightly enlarged $(1,630 \mathrm{~g}$.) and pale yellow. The oesophagus, stomach, and small and large intestine were normal.

No abnormality was found in the endocrine glands.

Histological findings The parasagittal abscess showed Gram-positive filaments and had a wall of thick fibrous tissue. The cerebral abscesses were filled with a chronic purulent exudate, the wall consisting of glial fibres and abundant astrocytes. Small abscesses containing Grampositive coccoid organisms were seen in the renal cortex.

Mitral and tricuspid vegetations consisted of clumps of fibrin mingled with clusters of oval coccoid microorganisms.

The red pulp of the spleen was moderately congested; sinusoids contained an excess of polymorphonuclear leucocytes and many haemosiderin deposits were seen. Haemosiderin was also present in moderately large amounts in the sections of liver. The marrow was of rather low cellularity with an excess of primitive cells but few late normoblasts; deposits of haemosiderin were abundant.

CASE 2 A man aged 67, a retired insurance manager, was first seen in November 1957 with 18 months' history of spontaneous bruising. Examination disclosed moderate enlargement of the liver and spleen but no lymphadenopathy.

On admission $\mathrm{Hb}$ was $10 \cdot 8 \mathrm{~g}$. per $100 \mathrm{ml}$. of blood, W.B.C. $9,800 /$ c.mm. (polymorphs 7,300, lymphocytes 1,400 , monocytes 540). Blood films showed a few late normoblasts and myelocytes in the peripheral blood. Platelets numbered $30,000 / \mathrm{c}$.mm. Hess's test was positive. The bleeding time (Ivy) was $\mathbf{1 0}$ minutes, and the clotting time four minutes. A left iliac crest biopsy showed normal marrow cells diluted with blood; further biopsy was postponed because of bleeding from the puncture site.

A provisional diagnosis of myelofibrosis was made and prednisone, $20 \mathrm{mg}$. t.d.s., started. Ten days later the platelets had risen to $120,000 / \mathrm{c} . \mathrm{mm}$. and the W.B.C. to $26,000 /$ c.mm. (polymorphs 21,000 ).

Prednisone $(5 \mathrm{mg}$. t.d.s.) was continued with the patient coming as an outpatient, and he continued well with only slight bruising until October 1959. During this time the W.B.C. rose gradually to 37,000 per c.mm. and platelets varied from 35,000 to $180,000 /$ c.mm.

He was readmitted in September 1959 with six weeks' history of a painful swelling of the left buttock which appeared to be an acute pyogenic abscess.

On this occasion $\mathrm{Hb}$ was $9.7 \mathrm{~g}$. per $100 \mathrm{ml}$. and $\overrightarrow{\vec{c}}$ W.B.C. numbered $60,000 / \mathrm{c} . \mathrm{mm}$. (polymorphs $43,000 \stackrel{0}{?}$ myelocytes 10,000 , lymphocytes 4,700 , monocytes 1,000 ). Platelets were $80,000 / \mathrm{c} . \mathrm{mm}$. The abscess was incised and흠. $8 \mathrm{oz}$. of thick yellow pus obtained from it. Sulphadimidine, $1 \mathrm{~g}$. four hourly, was started.

Cultures of the pus grew Nocardia. Postoperatively then patient's condition deteriorated; the $\mathrm{Hb}$ fell to $6.1 \mathrm{~g}$. per? $100 \mathrm{ml}$., urine output fell, and the blood urea rose to $\overrightarrow{0}$ $110 \mathrm{mg} . / 100 \mathrm{ml}$. He became semiconscious and died on $\overrightarrow{-}$ 10 October 1959. Permission for a necropsy was refused. $\mathscr{\sigma}_{\tilde{\sigma}}$

\section{BACTERIOLOGY}

MORPHOLOGY The pus obtained from the abscesses $\omega$ of both patients was thick, yellow, and odourless 5 Direct examination of films of the pus showed as filamentous Gram-positive organism which branched and stained irregularly. The organism was weaklys acid fast and on subculture tended to break up inte coccoid forms and show a beaded appearance.

CULTURAL CHARACTERISTICS Growth occurred ori most ordinary media, including nutrient and bloow agar, Sabouraud's medium, Lowenstein-Jensens slopes, nutrient and glucose broth. Of these bloop agar was most useful. All original cultures yielded कo pure growth. On blood agar incubated aerobically? minute grey colonies embedded in the medium weres

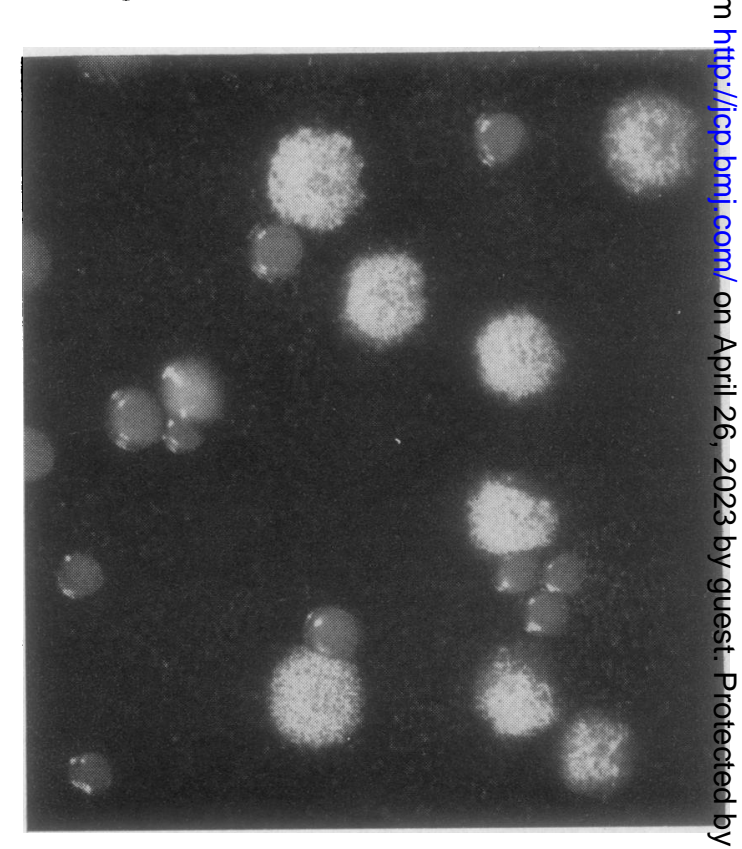

FIG. 1. Colonial variation of Nocardia asteroides. 
just visible in 24 hours. With continued incubation, for 48 to 96 hours, two types of colony could be distinguished: smoath, domed colonies 1-2 mm. diameter, and colonies of similar size with a rough powdery surface, pale yellow or white (Fig. 1). With continued incubation all colonies became taller and developed a rough surface. After incubation for a week the rough surface could be seen to be due to the development of aerial hyphae. Old cultures become deeply pigmented, usually red but occasionally yellow. Growth did not occur anaerobically. Cultures left on the bench grew quite well but growth was most rapid at $30-40^{\circ} \mathrm{C}$.

BIOCHEMICAL REACTIONS Urea was split on Christenson's media by both organisms. Slight and inconsistent fermentation occurred in peptone water sugars. In an attempt to obtain specific biochemical reactions the methods of Gordon and her colleagues (Gordon and Smith, 1955; Gordon and Mihm, 1957, 1959) using sugars incorporated in agar, containing inorganic salts only, were used. The results (Table I) were similar to those of Gordon and Mihm who tested 98 strains.

\section{TABLE I}

BIOCHEMICAL REACTIONS OF SPECIES OF NOCARDIA ISOLATED FROM CASES 1 AND 2

\begin{tabular}{|c|c|c|c|c|}
\hline & \multicolumn{2}{|c|}{$\begin{array}{l}\text { Case } 1 \\
\text { After Incubation }\end{array}$} & \multicolumn{2}{|c|}{$\begin{array}{l}\text { Case } 2 \\
\text { After Incubation }\end{array}$} \\
\hline & 8 Days & 28 Days & 8 Days & 28 Days \\
\hline \multicolumn{5}{|c|}{ Decomposition of: } \\
\hline Casein & - & - & - & - \\
\hline Gelatin & + & & - & - \\
\hline Tyrosine & - & - & - & - \\
\hline \multicolumn{5}{|l|}{ Acid from: } \\
\hline Arabinose & - & + & - & + \\
\hline Galactose & - & + & - & + \\
\hline Glucose & + & + & + & + \\
\hline Inositol & - & - & - & - \\
\hline Lactose & - & - & - & Weak + \\
\hline Maltose & - & Weak + & - & + \\
\hline Mannitol & - & - & - & - \\
\hline Raffinose & - & Weak + & - & - \\
\hline Rhamnose & + & + & - & - \\
\hline Sorbitol & - & - & - & - \\
\hline Xylose & - & - & - & - \\
\hline
\end{tabular}

ANTIBIOTIC SENSITIVITIES Strips of filter paper soaked in solutions of antibiotics were incorporated in nutrient agar plates for testing sensitivities. Organisms of known sensitivities were used as controls. Both organisms were resistant to penicillin and erythromycin and sensitive to chloramphenicol and sulphamezathine. The organism from Case 1 was resistant to streptomycin and tetracycline, that from Case 2 was sensitive to these two antibiotics.
SEROLOGICAL INVESTIGATIONS No agglutinating or complement-fixing antibodies could be found in sera from Case 1 using live and formalized suspensions of the organism.

\section{COMMENT ON BACTERIOLOGY}

The classification of the Actinomycetaceae is still unsettled. Waksman and Henrici (1943) divide the aerobic actinomycetaceae into Nocardia, in which the mycelium breaks up on subculture, and streptomycetae, in which the mycelium remains intact. Both organisms described in this paper fulfil this definition for Nocardia.

$N$. asteroides is the most common member of the genus; it is acid fast, whereas other Nocardia and Streptomycetae are not acid fast or only occasionally acid fast (Mariat, 1958), and give the biochemical reactions described by Gordon and Mihm. Both organisms are therefore provisionally identified as Nocardia asteroides.

ANIMAL PATHOGENICITY Rabbits and guinea-pigs were infected with the organisms from Cases 1 and 2 in order to study the type and distribution of the lesions produced and also to determine the effect of intramuscular prednisone on the course of such infections.

The organism was suspended in physiological saline producing an even suspension of opacity equivalent to Brown's tube 10. Rabbits were injected with $0.1 \mathrm{ml}$. intravenously. Groups of guinea-pigs received $0.1 \mathrm{ml}$. intracardially, under ether anaesthesia, or $0.1 \mathrm{ml}$. intraperitoneally, or $0.1 \mathrm{ml}$. intradermally. One batch of the last group was also given prednisone intraperitoneally in comparable doses to those used in man.

Rabbits died in about four days with elevation of the blood urea $(200 \mathrm{mg} . / 100 \mathrm{ml}$.) and those guinea-pigs infected by means of the intracardiac route died in about two days. Histological examination revealed multiple small abscesses composed largely of polymorphonuclear neutrophil leucocytes with a few histiocytes in the lungs, choroid plexus (Fig. 2), myocardium, liver, spleen, skeletal muscles, and in other tissues. Organisms were found with difficulty in the lesions, although cultured with ease. Often they appeared as chains of cocci, occasionally as short, Gram-positive, branching filaments. Filaments were more often seen in the more extensive lesions which tended to occur in the rabbits (Fig. 3). Splenic lesions were curious: Malpighian bodies were surrounded by histiocytes, themselves bordered by an area of fibrinoid necrosis containing polymorphonuclear neutrophil leucocytes but few microorganisms. 


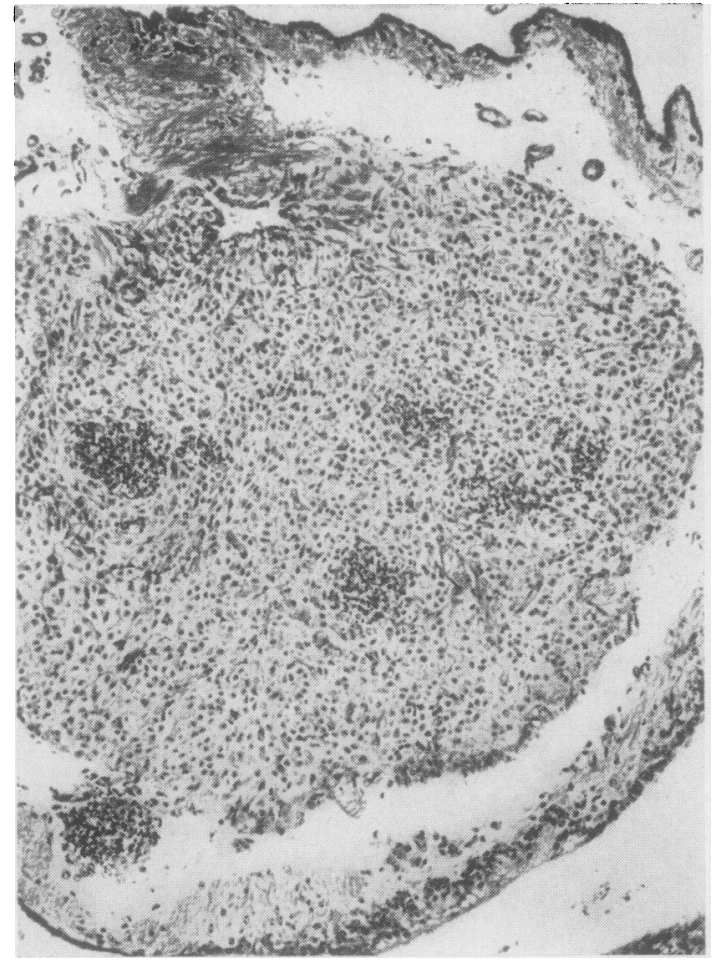

FIG. 2. A nocardial lesion in the tela choroidea of a rabbit (haematoxylin and eosin $\times 30$ ).

Intraperitoneal infection of guinea-pigs produced a similar result to that obtained by the intracardiac route. Lesions were, however, more abundant in the liver, mainly in portal tracts.

Intradermal infections remained localized in all but one animal where a few small lesions were ultimately found in the spleen when the animals were killed some weeks later. Prednisone had no effect on the progress of the disease. Indeed the animal showing splenic lesions after intradermal injection had not received the drug.

\section{DISCUSSION}

In the absence of pulmonary lesions in nocardiosis other portals of entry may be the gastrointestinal tract and superficial wounds (Ballenger and Goldring, 1957). There was no evidence of oral lesions in the two cases described in this paper and the intestinal tract of Case 1 was normal at necropsy. Nocardia are common soil organisms (Gordon and Hagan, 1936) and may cause localized infection in damaged tissues (Abbott, 1956); it is of interest that the appearance of the abscess in Case 2 was preceded by a fall. In Case 1 the patient was a hedger and had

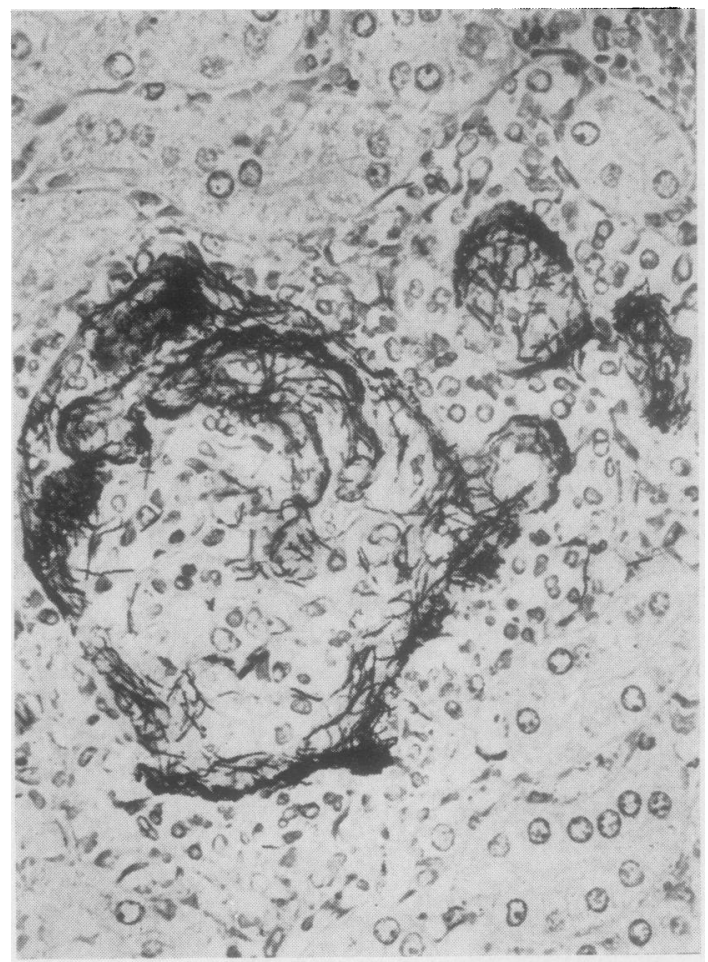

FIG. 3. Gram-positive filaments in the rabbit kidney (Gram stain $\times 400)$.

ample opportunities to acquire skin abrasions although no history of this was obtained.

Septicaemia following the development of localized 홍 abscesses is well recognized and Nocardia have been :isolated from the blood (Larsen, Diamond, and 3 . Collins, 1959). In Case 1 it seems likely that Nocardia were disseminated throughout the blood stream about two months before death, being localized in 0 the brain. Subsequently an endocarditis developed with metastatic abscesses in the kidneys. Bacteriological studies made after death were unhelpful; Nocardia was not isolated and only coliform bacillio and faecal streptococci were grown from a cardiac $N$ valve. The finding of Gram-positive filaments in N material from the parasagittal abscesses and failure $\sigma$ of cultures of this material to grow Nocardia suggests that this infection had been almost eradi- $\frac{C}{\varnothing}$ cated at the time of death.

Localization of the infection, with abscesses, in the brain has frequently been reported (Kaufman ${ }_{0}^{\circ}$ and Prieto, 1952; Kirby and McNaught, 1946; $\overrightarrow{\mathbb{D}}$ Larsen et al., 1959). The histological findings in $\frac{}{\mathrm{D}}$ nocardiosis are those of a non-specific chronic $\varrho$ purulent inflammatory process. Endocarditis due to Nocardia asteroides has been reported by Cruzo 
and Clancy (1952); they grew the organism from a heart valve and demonstrated rather atypical filaments in sections. The nature of the endocarditis in Case 1 remains uncertain.

The increase in fungal infections in Great Britain (Riddell, 1956) associated with the use of cytotoxic drugs and steroids may lead to more frequent recognition of nocardial infections. Larsen et al. (1959) reported seven cases of nocardial infection; five cases were of leukaemia or malignant disease and three were treated with steroids. The degree of anaemia was not reported but the authors comment on the possible predisposing effect of steroids and cytotoxic drugs.

Although animal experiments performed by us do not support this view with respect to steroids, it seems likely that in patients already anaemic such therapy could enable the infection to become established more easily.
We wish to thank the physicians of Addenbrooke's Hospital for permission to publish the case reports, Mr. S. T. Haslam for assistance with the bacteriology, and Mr. S. W. Patman for the photomicrographs.

\section{REFERENCES}

Abbott, P. (1956). Trans. roy. Soc. trop. Med. Hyg., 50, 11. Ballenger, C. N., and Goldring, D. (1957). J. Pediat., 50, 145. Cruz., P. T., and Clancy, C. F. (1952). Amer. J. Path., 28, 607. Gordon, R. E., and Hagan, W. A. (1936). J. infect. Dis., 59, 200. -, and Mihm, J. M. (1957). J. Bact., 73, 15.

- _- (1959). J. gen. Microbiol., 20, 129.

_, and Smith, M. M. (1955). J. Bact., 69, 147.

Kaufman, N., and Prieto, L. C. (1952). A.M.A., Arch. Path., 53, 379. Kirby, W. M. M., and McNaught, J. B. (1946). Arch. intern. Med., 78, 578.

Larsen, M. C., Diamond, H. D., and Collins, H. A. (1959). A.M.A. Arch. intern. Med., 103, 712.

Mariat, F. (1958). In Fungous Diseases and their Treatment, ed. R. W. Riddell and G. T. Stewart, p. 114. Butterworth, London.

McQuown, A. L. (1955). Amer. J. clin. Path., 25, 2.

Riddell, R. W. (1956). Brit. med. J., 2, 783.

Waksman, S. A., and Henrici, A. T. (1943). J. Bact., 46, 337. 